\title{
Effect of Perivascular Adipose Tissue on Arterial Adrenergic Contractions in Normotensive and Hypertensive Rats With High Fructose Intake
}

\author{
A. ZEMANČÍKOVÁ ${ }^{1}$, J. TÖRÖK ${ }^{1}$ \\ ${ }^{1}$ Institute of Normal and Pathological Physiology, Slovak Academy of Sciences, Bratislava, Slovak \\ Republic
}

Received March 24, 2017

Accepted September 16, 2017

\begin{abstract}
Summary
The aim of this study was to investigate the effect of high fructose intake associated with moderate increase in adiposity on rat arterial adrenergic responses and their modulation by perivascular adipose tissue (PVAT). After eight-week-lasting substitution of drinking water with $10 \%$ fructose solution in adult normotensive Wistar-Kyoto rats (WKY) and spontaneously hypertensive rats (SHR), their systolic blood pressure, plasma triglycerides, and relative liver weight were elevated when compared to their respective control groups. Moreover, in SHR, body weight and relative heart weight were increased after treatment with fructose. In superior mesenteric arteries, PVAT exerted inhibitory influence on adrenergic contractile responses and this effect was markedly stronger in control WKY than in SHR. In fructose-administered WKY, arterial adrenergic contractions were substantially reduced in comparison with the control group; this was caused mainly by enhancement of anticontractile action of PVAT. The diminution of the mesenteric arterial contractions was not observed after fructose treatment in SHR. We conclude that the increase in body adiposity due to fructose overfeeding in rats might have prohypertensive effect. However, in WKY it might cause PVAT-dependent and independent reduction in arterial contractile responses to adrenergic stimuli, which could attenuate the pathological elevation in vascular tone.
\end{abstract}

\section{Key words}

Perivascular adipose tissue - Rat mesenteric artery • Fructose • Hypertension • Adrenergic contraction

\section{Corresponding author}

A. Zemančíková, Institute of Normal and Pathological Physiology,
Slovak Academy of Sciences, Sienkiewiczova 1, 81371 Bratislava, Slovak Republic. E-mail: anna.zemancikova@savba.sk

\section{Introduction}

Increased values of body mass index (BMI) achieving the level of obesity are generally considered to associate with higher cardiovascular risk due to cardiac overload, activation of pressor systems, and diabetic impairment of heart and blood vessels (Vaněčková et al. 2014, Head 2015). However, the results of some recent studies have brought indications that moderately increased body fat might even be connected with better survival in patients with documented cardiovascular disease. Azimi et al. (2013) demonstrated that moderate overweight (BMI 27.5-30) positively influences survival in subjects with coronary heart disease. Furthermore, authors from all over the world confirmed such effect in overweight/pre-obese persons and this phenomenon was termed - and probably a little overstated - as "obesity paradox" (Curtis et al. 2005, Kumakura et al. 2010).

The link between increased adiposity and modification in cardiovascular function could also comprise the adipose tissue which surrounds and is closely adjacent to heart and vessels. This adipose compartment, previously considered as having only mechanical and isolating function, is now regarded as a highly metabolically active organ, which directly influences the functional and other properties of the adjoining cardiovascular tissues. It was shown, e.g. that perivascular adipose tissue (PVAT) attenuates 
vasoconstriction in many types of vessels; this was demonstrated mainly in rat conduit and small arteries (Soltis and Cassis 1991, Verlohren et al. 2004). It is suggested that under physiological conditions, healthy perivascular adipocytes produce as yet unidentified transferable factor(s) termed adipocyte-derived relaxing factor (ADRF) which hyperpolarizes vascular smooth muscle cells by activating different types of potassium channels on their membrane, and this process leads to inhibition of vasoconstriction (Gollash 2012, Tano et al. 2014). A number of PVAT-derived "anticontractile" molecules have been identified so far; besides adipokines (e.g. adiponectin), these include also cytokines/ chemokines, some gaseous molecules (nitric oxide, hydrogen sulphide), angiotensin 1-7, methyl palmitate, and reactive oxygen species (hydrogen peroxide) (Gao et al. 2007, Lee et al. 2011, Szasz and Webb 2012, Cacanyiova et al. 2016). However, there are many evidences that in pathological conditions associated with cardiovascular and metabolic dysfunction, PVAT could contribute to adverse alterations in vessel morphology and function, by releasing substances that support inflammation, smooth muscle cell migration, and atherogenesis (Uchida et al. 2016, Xia and Li 2017). It was shown that in obese patients with metabolic syndrome the total PVAT mass around small arteries was increased while its anticontractile effect was completely lost, and markers of hypoxia and inflammation were detected (Greenstein et al. 2009). Similar results were found also in animal genetic and diet-induced models of obesity (Ketonen et al. 2010, Aghamohammadzadeh et al. 2015). On the other hand, in spontaneously hypertensive rats (SHR) characteristic by low body weight (Puzserova et al. 2014), the defect in anticontractile action of mesenteric arterial PVAT was also detected and is proven to be importantly involved in the exaggerated sensitivity of SHR arteries to vasoconstrictors (Gálvez et al. 2006, Török et al. 2016). The potential causal relationship between the reduced PVAT mass and its insufficient anticontractile effect in SHR might support the idea about the above-mentioned paradox of beneficial impact of moderately increased body fat content on cardiovascular system.

The aim of this study was to investigate the effect of moderate growth in adiposity in normotensive and spontaneously hypertensive rats on anticontractile effect of PVAT in their mesenteric arteries. For this purpose, long-term high fructose administration was applied to rats because this monosaccharide has specific lipogenic metabolism and it is capable to evoke body weight gain (Lustig 2010, Tappy and Lê 2010).

\section{Methods}

The animal protocols were performed in accordance with the Guide for the Care and Use of Laboratory Animals published by the National Institutes of Health, and they were approved by the Animal Health and Welfare Division of the State Veterinary and Food Administration of the Slovak Republic. Twelve-week-old male normotensive Wistar-Kyoto rats (WKY) and spontaneously hypertensive rats were used and randomly divided into following groups: WKY $(n=10)$ and SHR $(n=9)$ receiving fructose (as $10 \%$ solution replacing drinking water) for the period of the following eight weeks, and the age-matched control WKY $(n=7)$ and SHR ( $n=7)$ receiving just tap water. All rats were housed at $22-24{ }^{\circ} \mathrm{C}$ on a $12: 12$-h dark-light cycle (06.00 - $18.00 \mathrm{~h}$ lights on) and maintained on a standard laboratory rat chow ad libitum.

Systolic blood pressure was measured in conscious animals by the non-invasive tail-cuff method. At the end of the treatment (i.e. in $20^{\text {th }}$ week of life), rats were sacrificed under $\mathrm{CO}_{2}$ anesthesia, their relative heart weights and relative liver weights (as the ratios of heart or liver weight to tibia length) were determined and samples of their blood were collected for measurement of plasma triglyceride concentration.

The superior mesenteric arteries were isolated from all rats and collected in cold modified Krebs solution with the following composition (in mmol/l): $\mathrm{NaCl} 118$, $\mathrm{KCl} 5, \mathrm{CaCl}_{2}$ 2.5, $\mathrm{MgSO}_{4} 1.2, \mathrm{KH}_{2} \mathrm{PO}_{4}$ 1.2, $\mathrm{NaHCO}_{3} 25$, glucose $11, \mathrm{CaNa}_{2}$ EDTA 0.03 . The arteries were cut into rings $3.0-3.5 \mathrm{~mm}$ of length. Paired arterial rings, one with PVAT preserved and the other with PVAT removed, were prepared from each artery. PVAT removal was carried out with fine scissors under microscope with extra caution not to damage the adventitial layer. The arteries were suspended in $20 \mathrm{ml}$ organ chambers with oxygenated $\left(95 \% \mathrm{O}_{2}+5 \% \mathrm{CO}_{2}\right.$ ) modified Krebs solution maintained at $37^{\circ} \mathrm{C}$. For measurement of contractile activity, the preparations were set up for isometric tension recording using a force-displacement transducer Sanborn FT 10 (Sanborn, Baltimore, USA). The preparations were equilibrated under a resting tension of $10 \mathrm{mN}$ for 60 - $90 \mathrm{~min}$, and the solution was changed every $15 \mathrm{~min}$.

Adrenergic contractions were determined in endothelium-intact mesenteric arteries, using the same 
protocol in rings with intact PVAT and in rings with PVAT removed. First, neurogenic responses were elicited by electrical stimulation of periarterial sympathetic nerves. The arterial rings were stimulated by two parallel platinum plate electrodes placed on either side of the preparation and connected to an electrostimulator ST-3 (Hungary). Frequency-response curves to electrical stimuli were obtained using square pulses of $0.5 \mathrm{~ms}$ in duration, at supramaximal voltage $(>30 \mathrm{~V})$, applied at $1-32 \mathrm{~Hz}$ for a period of $20 \mathrm{~s}$. The neurogenic (sympatho-adrenergic) origin of the contractile responses was confirmed pharmacologically, as described in Török and Zemančíková (2016), indicating that they were elicited mainly by endogenous noradrenaline released from electrically stimulated periarterial adrenergic nerves. Subsequently, after $30 \mathrm{~min}$ of recovery, the concentration-dependent contractile responses to cumulatively applied exogenous noradrenaline were performed. At the end of the measurements, contraction to excitation by high $\mathrm{K}^{+}$concentration in bath solution $(100 \mathrm{mmol} / \mathrm{l} \mathrm{KCl})$ was determined in each artery.

The chemicals used were purchased from Sigma-Aldrich (Germany). All drugs were dissolved in distilled water and concentration was expressed as final concentration in the incubation chamber.

Arterial isometric contractile responses to particular stimuli were expressed as the active wall tension in $\mathrm{mN}$ and normalized to the length of respective ring preparation $(\mathrm{mm})$. Area under curve (AUC, in arbitrary units) was calculated from individual concentration/frequency-response curves in each experimental group. The negative $\log _{10}$ concentration required to achieve the half-maximum contraction $\left(\mathrm{pEC}_{50}\right)$ was calculated for each dose-response curve to express the sensitivity to noradrenaline.
The results are presented as means $\pm \mathrm{SEM}$. Statistical evaluation was carried out by using Student's unpaired t-test. The differences were considered to be significant when $p<0.05$.

\section{Results}

The values in the Table 1 show that in $20^{\text {th }}$ week of life, control SHR had higher blood pressure, heart rate and relative heart weight; however, their body weight was significantly lower compared to that in control WKY. Administration of fructose from $12^{\text {th }}$ to $20^{\text {th }}$ week of life caused increase in systolic blood pressure in WKY as well as in SHR, without change in their heart rate. However, in SHR, higher values of relative heart weight were found after fructose feeding. Both fructose-fed groups had higher levels of plasma triglycerides and relative liver weights when compared to their respective controls. Body weight was increased due to fructose administration only in SHR.

In mesenteric arteries from control WKY, the presence of PVAT caused reduction in contractile responses to exogenous noradrenaline (AUC; Table 2) as well as to endogenous noradrenaline released from excited perivascular sympathetic nerves during transmural electrical stimulation (TES); however, in response to TES, the decrease due to PVAT was detected only at lower frequencies of stimulation $(1-4 \mathrm{~Hz})(p<0.01)$ and did not manifest significantly in AUC values (Table 2). In WKY arteries with intact PVAT, the sensitivity in dosedependent contractile responses to exogenous noradrenaline (expressed as $\mathrm{pEC}_{50}$ ) was also decreased in comparison to that in arteries with removed PVAT. The contractions induced by $100 \mathrm{mmol} / \mathrm{l} \mathrm{KCl}$ were not influenced by the presence of PVAT (Table 2).

Table 1. General characteristics of experimental animals.

\begin{tabular}{lcccc}
\hline & WKY & WKYfru & SHR & SHRfru \\
\hline$B W(\mathrm{~g})$ & $422.24 \pm 11.70$ & $430.20 \pm 8.67$ & $322.60 \pm 6.90^{+++}$ & $353.00 \pm 7.45 *$ \\
$S B P(\mathrm{~mm} \mathrm{Hg})$ & $114.69 \pm 2.13$ & $121.06 \pm 1.56^{*}$ & $200.28 \pm 7.57^{+++}$ & $222.13 \pm 3.61 *$ \\
$H R(\mathrm{bpm})$ & $329.19 \pm 6.74$ & $334.25 \pm 6.61$ & $482.43 \pm 7.22^{+++}$ & $509.67 \pm 21.39$ \\
$H W / T L(\mathrm{mg} / \mathrm{mm})$ & $31.41 \pm 0.55$ & $30.45 \pm 0.69$ & $35.17 \pm 0.54^{++}$ & $39.58 \pm 1.13 * *$ \\
$L W / T L(\mathrm{mg} / \mathrm{mm})$ & $260.90 \pm 8.67$ & $295.41 \pm 11.26^{*}$ & $244.90 \pm 7.40$ & $275.18 \pm 4.85 * *$ \\
$T R G L(\mathrm{mmol} / \mathrm{l})$ & $0.68 \pm 0.07$ & $1.06 \pm 0.11 * *$ & $0.52 \pm 0.02$ & $0.67 \pm 0.02 * *$ \\
\hline
\end{tabular}

BW - body weight, SBP - systolic blood pressure, HR - heart rate, HW/TL - heart weight/tibia length, LW/TL - liver weight/tibia length, TRIGL - plasma triglycerides. Values represent mean $\pm \mathrm{SEM} ; \mathrm{n}=7-10 . * p<0.05, * * p<0.01$ fructose-treated vs. respective control rats; ${ }^{++} p<0.01,{ }^{+++} p<0.001$ control SHR vs. WKY. 
Table 2. Comparison of contractile characteristics in mesenteric arteries with perivascular adipose tissue removed (PVAT-) and intact (PVAT+), obtained from control and fructose-treated (fru) normotensive Wistar-Kyoto rats (WKY) and spontaneously hypertensive rats (SHR).

\begin{tabular}{|c|c|c|c|c|c|}
\hline & & \multicolumn{2}{|c|}{$\mathbf{N A}$} & \multirow{2}{*}{$\frac{\text { TES }}{\text { AUC }}$} & \multirow{2}{*}{$\begin{array}{c}100 \text { mmol/l KCl } \\
(\mathrm{mN} / \mathrm{mm})\end{array}$} \\
\hline & & $\mathrm{pEC}_{50}$ & $\mathbf{A U C}$ & & \\
\hline \multirow{2}{*}{ WKY } & PVAT- & $6.50 \pm 0.06$ & $17.06 \pm 1.04$ & $3.68 \pm 0.28$ & $2.10 \pm 0.19$ \\
\hline & PVAT + & $5.40 \pm 0.09 * * *$ & $6.09 \pm 0.76^{* * *}$ & $3.00 \pm 0.43$ & $2.05 \pm 0.21$ \\
\hline \multirow{2}{*}{ WKYfru } & PVAT- & $6.34 \pm 0.08$ & $14.08 \pm 0.85$ & $3.13 \pm 0.29$ & $2.03 \pm 0.20$ \\
\hline & PVAT + & $4.94 \pm 0.12 * * *$ & $3.27 \pm 0.54 * * *$ & $2.39 \pm 0.38$ & $1.79 \pm 0.16$ \\
\hline \multirow{2}{*}{ SHR } & PVAT- & $6.35 \pm 0.13$ & $16.79 \pm 0.79$ & $7.28 \pm 0.95$ & $2.96 \pm 0.20$ \\
\hline & PVAT + & $6.04 \pm 0.15$ & $12.16 \pm 1.82 *$ & $8.60 \pm 1.42$ & $2.86 \pm 0.17$ \\
\hline \multirow{2}{*}{ SHRfru } & PVAT- & $6.29 \pm 0.08$ & $18.16 \pm 1.13$ & $8.71 \pm 0.84$ & $3.43 \pm 0.13$ \\
\hline & PVAT+ & $5.87 \pm 0.15 *$ & $14.57 \pm 1.11 *$ & $9.62 \pm 0.68$ & $3.54 \pm 0.27$ \\
\hline
\end{tabular}

NA - noradrenaline; TES - transmural electrical stimulation; $\mathrm{pEC}_{50}$ - negative logarithm of half maximal effective noradrenaline concentration; AUC - area under curve; $\mathrm{KCl}$ - potassium chloride. Values represent mean $\pm \mathrm{SEM} ; \mathrm{n}=7-10$. $* p<0.05, * * * p<0.001$ PVAT+ vs. PVAT- in the respective experimental group of rats.

Contractile responses to exogenous noradrenaline were significantly higher in PVAT-intact arteries from control SHR comparing to WKY, which is demonstrated also by higher sensitivity $\left(\mathrm{pEC}_{50}\right)$ and AUC in SHR; however, the differences between SHR and WKY arterial contractions were absent in preparations without PVAT (Table 2). Similarly to WKY, the reduction in noradrenaline contractile responses due to the preservation of PVAT on arterial surface was observed in control SHR (Fig. 1), which was manifested also by smaller AUC (Table 2); however, the anticontractile effect of PVAT in SHR was evidently weaker when comparing to WKY. The arterial neurogenic contractions evoked by TES were significantly higher in control SHR in comparison with WKY arteries, both with or without PVAT. The diminishing effect of PVAT on mesenteric arterial contractions to TES and to $100 \mathrm{mmol} / \mathrm{l} \mathrm{KCl}$ was not detected in SHR (Table 2).

Figure 1A illustrates the reduction in contractile responses to exogenous noradrenaline in WKY mesenteric arteries after fructose administration. The diminution of contraction due to fructose was manifested in both types of preparations - with PVAT intact as well as PVAT removed; however, it was more pronounced in arteries with preserved PVAT. In contrast, the fructose-induced anticontractile effect was not seen in SHR arteries; the only change detected after fructose administration was the increase in contraction in response to the highest noradrenaline concentration in PVAT-intact arteries (Fig. 1B).

When considering the neurogenic contractions to TES, the effect of fructose treatment was detected only in WKY arteries without PVAT; in these preparations, the contractile responses were significantly smaller in comparison with arteries from control WKY. In SHR, fructose administration did not induce any changes in arterial responses to EFS (Fig. 2).

Contractions induced by $100 \mathrm{mmol} / 1 \mathrm{KCl}$ were not changed due to fructose administration (Table 2).

\section{Discussion}

In this study we have confirmed that in healthy individuals, PVAT exhibits mostly inhibitory effect on arterial contraction which is in agreement with the results of other authors showing anticontractile action of this tissue in physiological conditions without significant manifestation of inflammatory and proliferative processes (Szasz and Webb 2012). Moreover, our findings indicate that in healthy rats moderate increase in adiposity induced by high fructose intake might be associated with enhancement of anticontractile effect of PVAT on arterial wall. This could be mediated by the increase and/or change in spectrum of substances with relaxant properties which are released from perivascular adipocytes and hereby contribute to attenuation of possible increase in arterial tension. 
A

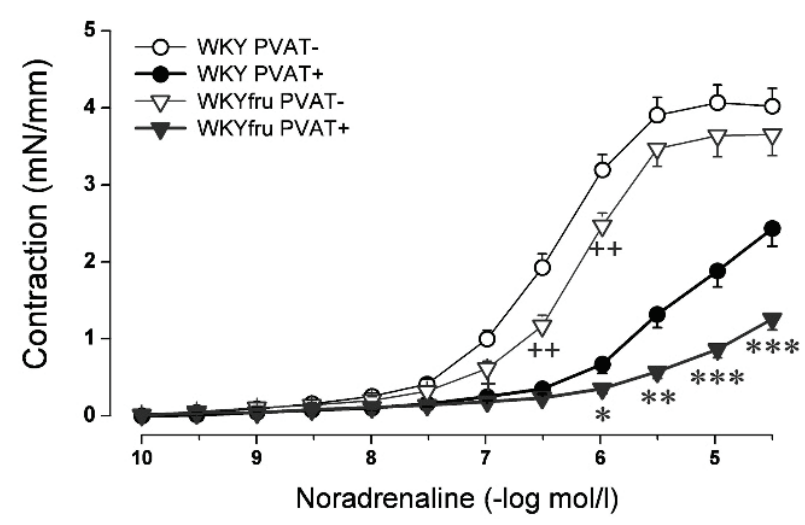

B

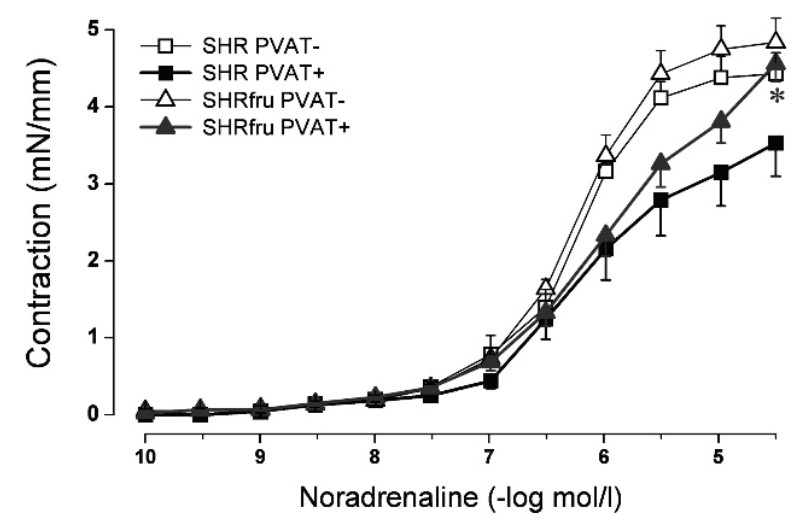

Fig. 1. Effect of eight-week-lasting fructose administration in normotensive Wistar-Kyoto rats (WKY, A) and spontaneously hypertensive rats (SHR, B) on dose-dependent contractile responses to exogenous noradrenaline in superior mesenteric arteries with PVAT intact (+) and removed (-). Values represent mean $\pm \mathrm{SEM} ; \mathrm{n}=7-10 ; * p<0.05, * * p<0.01, * * * p<0.001$ PVAT+ artery of fructose-treated vs. control WKY/SHR; ${ }^{+} p<0.05,{ }^{++} p<0.01$ PVAT- artery of fructose-treated vs. control WKY.

A

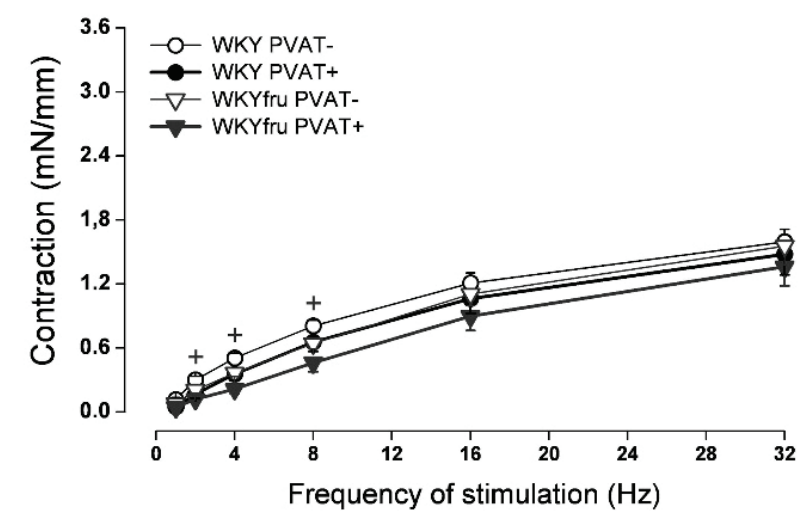

B

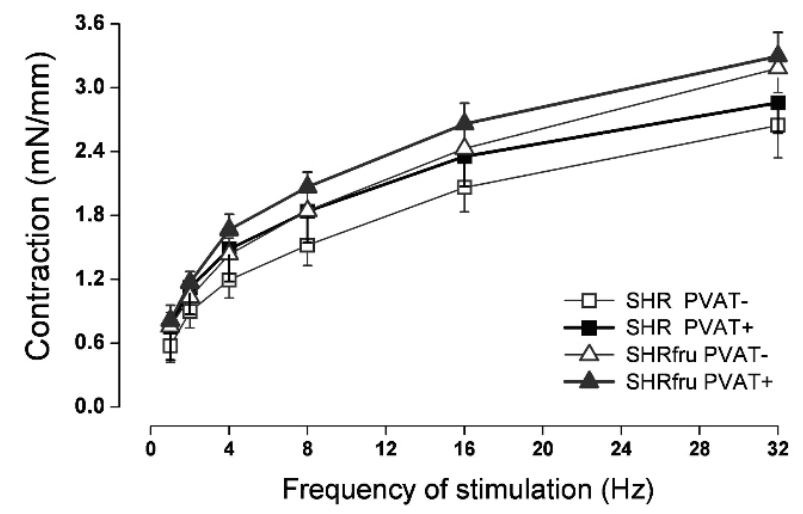

Fig. 2. Effect of eight-week-lasting fructose administration in normotensive Wistar-Kyoto rats (WKY, $\mathbf{A})$ and spontaneously hypertensive rats $(\mathrm{SHR}, \mathbf{B})$ on frequency-dependent neurogenic contractile responses to transmural electrical stimulation in superior mesenteric arteries with PVAT intact $(+)$ and removed $(-)$. Values represent mean \pm SEM; $n=7-10 ;{ }^{+} p<0.05$ PVAT- artery of fructose-treated vs. control WKY.

Fructose is a highly lipogenic sugar that has profound metabolic effects in the liver and its excessive consumption in humans has been associated with the components of metabolic syndrome (insulin resistance, elevated waist circumference, dyslipidemia, and hypertension) (Dekker et al. 2010, Tappy and Lê 2010). It was shown that rats fed with fructose in the long-term develop hepatic steatosis, increase in adipose tissue mass and in circulating triglycerides, and decreased sensitivity to insulin and leptin (Lustig 2010). In our experiments, after eight weeks of fructose administration, WKY exhibited significantly increased liver weight and elevated plasma triglycerides indicating changes in hepatic metabolism of lipids. It can be assumed that some alterations might have been triggered also in adipocytes, due to increased circulating lipids and the necessity of their storage, which could lead to the growth of adipose tissue mass. Similar results were published by Crescenzo et al. (2014) who found that fructose-fed WKY had higher weight of particular adipose deposits (mesenteric, epididymal) in spite of unchanged total body weight, when compared to control group. The results presented in this study indicate that such trophic changes might have concerned also PVAT, and that they could be manifested initially by potentiating of its anticontractile properties.

In contrast, many authors demonstrated that in conditions of obesity and metabolic syndrome in humans as well as in experimental animals, PVAT has a potential to become "dysfunctional" in the sense that it contributes to vascular dysfunction through the release of 
pro-contractile, atherogenic, proliferatory and pro-migratory substances. Such adverse properties of PVAT could be manifested by its altered vasomodulatory function and might profoundly alter the function of particular components of the vessel wall in the long-term (Greenstein et al. 2009, Ketonen et al. 2010, Aghamohammadzadeh et al. 2015).

Regarding the presented results from fructoseadministered rats, one can presume that later, after progression in metabolic syndrome development, accompanied with further increase in adiposity and with expression of diabetic impairments in these rats, the character of PVAT action would change and it would become pro-contractile and pro-inflammatory. This is in agreement with the results presented by Beltowski (2013) who found that feeding rats with "cafeteria" (high-calorie) diet for 3 months resulted in obesity with signs of metabolic syndrome, whereas 1 month of feeding led to adiposity without insulin resistance. Interestingly, 1 month-lasting "cafeteria" diet increased production of hydrogen sulphide by PVAT and this was associated with an enhanced anticontractile effect of PVAT, whereas long-term "cafeteria" diet reduced hydrogen sulphide production and anticontractile effects of PVAT (Beltowski 2013).

With regard to the above-mentioned facts one can state that to the certain level of its growth, PVAT may exhibit protective and beneficial action on vascular function. It seems that this "positive" effect might probably be caused non-specifically, produced only as a consequence of growing PVAT mass and the respective increase in production of vasorelaxant factors (predominant effect of quantity). However, after reaching certain level of adiposity, the properties of PVAT could importantly change due to inflammatory and diabetic alterations of this tissue. This is in agreement with the schema of Xia and Li (2017) who published an idea about the central mechanisms underlying the PVAT dysfunction in obesity. They described this phenomenon as "obesity triad" consisting of PVAT hypoxia, inflammation and oxidative stress (Xia and Li 2017).

It should be mentioned that the attenuation of adrenergic contractile responses observed in fructosetreated WKY could not be attributed only to PVAT because arterial preparations without PVAT also exhibited smaller contractions to exogenous noradrenaline and to TES. The anticontractile mechanisms (PVAT-dependent and independent) in fructose-administered WKY did not prevent the slight but significant increase in blood pressure; however, one can suppose that they could importantly slow-down it.

The situation seems to be different in SHR; this genetically hypertensive rat strain is characteristic by lower body weight and corresponding smaller body fat content in comparison with control WKY. Gálvez et al. (2006) found that the mesentery weight and its total lipid content are lower in SHR than in WKY. In this study we have confirmed that comparing to WKY, the anticontractile effect of PVAT in mesenteric arteries of SHR is significantly smaller, as shown also by other authors (Gálvez et al. 2006, Li et al. 2013). Therefore, one can speculate that the relationship between body adiposity and anticontractile properties of PVAT creates an analogy to the findings of Azimi et al. (2013) who described the subjects with lower BMI as having higher risk of mortality associated with cardiovascular dysfunction. Lee et al. (2011) documented that the release of the relaxant substance - palmitic acid methyl ester - is decreased in PVAT from SHR arteries; in contrast, PVAT production of angiotensin II is higher in SHR compared to WKY. Lu et al. (2011) showed that PVAT in thoracic aorta from SHR had similar cross section area compared to WKY but it contained higher density and total number of brown adipocytes, which indicates that it could have poorer secretory capacity. Except the decreased production of PVAT-derived relaxing factors, it was proposed that higher sympathetic innervation in PVAT (Török et al. 2016) and the deficient function of potassium channels in vascular smooth muscle (Li et al. 2013, Bencze et al. 2016) could also partially contribute to the weaker anticontractile effect of PVAT in arteries from SHR. Gálvez-Prieto et al. (2008) documented that most of the above-mentioned PVAT alterations in SHR are manifested in $4^{\text {th }}$ week of their age and precede the development of hypertension, suggesting that they are strongly fixed in SHR phenotype. This could partially explain our findings that fructoseinduced moderate increase in body weight (and probably also in adiposity) did not induce significant changes in PVAT modulation of adrenergic responses in mesenteric artery of SHR. One can speculate that in SHR the paradox of beneficial effect of moderate increase in adiposity does not exist and that the growth in adipose tissue would continually pass to further impairment of arterial function.

In conclusion, the relationship between quantitative and qualitative characteristics of PVAT is not 
definite. Similarly to the paradoxical beneficial effect of overweight on cardiovascular function in humans, it seems that moderate growth in adipose tissue induced by high fructose administration could enhance the anticontractile action of PVAT in normotensive WKY rats which might attenuate the pathological increase in vascular tone during gaining weight. In contrast, in SHR which exhibit substantial impairments of arteries and of their regulation by PVAT in control conditions, the fructose-induced increase in adiposity does not lead to the improvement of PVAT function and to the reduction in arterial sensitivity to adrenergic stimuli.

\section{Conflict of Interest}

There is no conflict of interest.

\section{Acknowledgements}

The study was supported by VEGA grant No. 2/0202/17.

\section{References}

AGHAMOHAMMADZADEH R, UNWIN RD, GREENSTEIN AS, HEAGERTY AM: Effects of obesity on perivascular adipose tissue vasorelaxant function: nitric oxide, inflammation and elevated systemic blood pressure. J Vasc Res 52: 299-305, 2015.

AZIMI A, CHARLOT MG, TORP-PEDERSEN C, GISLASON GH, KØBER L, JENSEN LO, THAYSSEN P, RAVKILDE J, TILSTED HH, LASSEN JF, THUESEN L: Moderate overweight is beneficial and severe obesity detrimental for patients with documented atherosclerotic heart disease. Heart 99: 655-660, 2013.

BEŁTOWSKI J: Endogenous hydrogen sulfide in perivascular adipose tissue: role in the regulation of vascular tone in physiology and pathology. Can J Physiol Pharmacol 91: 889-898, 2013.

BENCZE M, BEHULIAK M, VAVŘÍNOVÁ A, ZICHA J: Altered contractile responses of arteries from spontaneously hypertensive rat: The role of endogenous mediators and membrane depolarization. Life Sci 166: 46-53, 2016.

CACANYIOVA S, BERENYIOVA A, KRISTEK F: The role of hydrogen sulphide in blood pressure regulation. Physiol Res 65 (Suppl 3): S273-S289, 2016.

CRESCENZO R, BIANCO F, COPPOLA P, MAZZOLI A, VALIANTE S, LIVERINI G, IOSSA S: Adipose tissue remodeling in rats exhibiting fructose-induced obesity. Eur J Nutr 53: 413-419, 2014.

CURTIS JP, SELTER JG, WANG Y, RATHORE SS, JOVIN IS, JADBABAIE F, KOSIBOROD M, PORTNAY EL, SOKOL SI, BADER F, KRUMHOLZ HM: The obesity paradox: body mass index and outcomes in patients with heart failure. Arch Intern Med 165: 55-61, 2005.

DEKKER MJ, SU Q, BAKER C, RUTLEDGE AC, ADELI K: Fructose: a highly lipogenic nutrient implicated in insulin resistance, hepatic steatosis, and the metabolic syndrome. Am J Physiol Endocrinol Metab 299: E685-E694, 2010.

GAO YJ, LU C, SU LY, SHARMA AM, LEE RM: Modulation of vascular function by perivascular adipose tissue: the role of endothelium and hydrogen peroxide. Br J Pharmacol 151: 323-331, 2007.

GÁlVEZ B, DE CASTRO J, HEROLD D, DUBROVSKA G, ARRIBAS S, GONZÁLEZ MC, ARANGUEZ I, LUFT FC, RAMOS MP, GOLLASCH M, FERNÁNDEZ ALFONSO MS: Perivascular adipose tissue and mesenteric vascular function in spontaneously hypertensive rats. Arterioscler Thromb Vasc Biol 26: 1297-1302, 2006.

GÁlVEZ-PRIETO B, DUBROVSKA G, CANO MV, DELGADO M, ARANGUEZ I, GONZÁlEZ MC, RUIZGAYO M, GOLLASCH M, FERNÁNDEZ-ALFONSO MS: A reduction in the amount and anti-contractile effect of periadventitial mesenteric adipose tissue precedes hypertension development in spontaneously hypertensive rats. Hypertens Res 31: 1415-1423, 2008.

GOLLASCH M: Vasodilator signals from perivascular adipose tissue. Br J Pharmacol 165: 633-642, 2012.

GREENSTEIN AS, KHAVANDI K, WITHERS SB, SONOYAMA K, CLANCY O, JEZIORSKA M, LAING I, YATES AP, PEMBERTON PW, MALIK RA, HEAGERTY AM: Local inflammation and hypoxia abolish the protective anticontractile properties of perivascular fat in obese patients. Circulation 119: 1661-1670, 2009.

HEAD GA: Cardiovascular and metabolic consequences of obesity. Front Physiol 6: 32, 2015.

KETONEN J, SHI J, MARTONEN E, MERVAALA E: Periadventitial adipose tissue promotes endothelial dysfunction via oxidative stress in diet-induced obese C57B1/6 mice. Circ J 74: 1479-1487, 2010. 
KUMAKURA H, KANAI H, AIZAKI M, MITSUI K, ARAKI Y, KASAMA S, IWASAKI T, ICHIKAWA S: The influence of the obesity paradox and chronic kidney disease on long-term survival in a Japanese cohort with peripheral arterial disease. J Vasc Surg 52: 110-117, 2010.

LEE YC, CHANG HH, CHIANG CL, LIU CH, YEH JI, CHEN MF, CHEN PY, KUO JS, LEE TJ: Role of perivascular adipose tissue-derived methyl palmitate in vascular tone regulation and pathogenesis of hypertension. Circulation 124: 1160-1171, 2011.

LI R, ANDERSEN I, ALEKE J, GOLUBINSKAYA V, GUSTAFSSON H, NILSSON H: Reduced anti-contractile effect of perivascular adipose tissue on mesenteric small arteries from spontaneously hypertensive rats: role of Kv7 channels. Eur J Pharmacol 698: 310-315, 2013.

LU C, SU LY, LEE RM, GAO YJ: Alterations in perivascular adipose tissue structure and function in hypertension. Eur J Pharmacol 656: 68-73, 2011.

LUSTIG RH: Fructose: metabolic, hedonic, and societal parallels with ethanol. J Am Diet Assoc 110: 1307-1321, 2010.

PUZSEROVA A, ILOVSKA V, BALIS P, SLEZAK P, BERNATOVA I: Age-related alterations in endothelial function of femoral artery in young SHR and WKY rats. Biomed Res Int 2014: 658479, 2014.

SOLTIS EE, CASSIS LA: Influence of perivascular adipose tissue on rat aortic smooth muscle responsiveness. Clin Exp Hypertens A 13: 277-296, 1991.

SZASZ T, WEBB RC: Perivascular adipose tissue: more than just structural support. Clin Sci (Lond) 122: 1-12, 2012.

TANO JY, SCHLEIFENBAUM J, GOLLASCH M: Perivascular adipose tissue, potassium channels, and vascular dysfunction. Arterioscler Thromb Vasc Biol 34: 1827-1830, 2014.

TAPPY L, LÊ KA: Metabolic effects of fructose and the worldwide increase in obesity. Physiol Rev 90: 23-46, 2010.

TÖRÖK J, ZEMANČÍKOVÁ A: Agmatine Modulation of noradrenergic neurotransmission in isolated rat blood vessels. Chin J Physiol 59: 131-138, 2016.

TÖRÖK J, ZEMANČÍKOVÁ A, KOCIANOVÁ Z: Interaction of perivascular adipose tissue and sympathetic nerves in arteries from normotensive and hypertensive rats. Physiol Res 65 (Suppl 3): S391-S399, 2016.

UCHIDA Y, UCHIDA Y, SHIMOYAMA E, HIRUTA N, KISHIMOTO T, WATANABE S: Pericoronary adipose tissue as storage and supply site for oxidized low-density lipoprotein in human coronary plaques. PLoS One 11: e0150862, 2016.

VANĚČKOVÁ I, MALETÍNSKÁ L, BEHULIAK M, NAGELOVÁ V, ZICHA J, KUNEŠ J: Obesity-related hypertension: possible pathophysiological mechanisms. J Endocrinol 223: R63-R78, 2014.

VERLOHREN S, DUBROVSKA G, TSANG SY, ESSIN K, LUFT FC, HUANG Y, GOLLASCH M: Visceral periadventitial adipose tissue regulates arterial tone of mesenteric arteries. Hypertension 44: 271-276, 2004.

XIA N, LI H: The role of perivascular adipose tissue in obesity-induced vascular dysfunction. Br J Pharmacol 174: 3425-3442, 2017. 